\title{
Switching to preservative-free latanoprost: impact on tolerability and patient satisfaction
}

\author{
This article was published in the following Dove Press journal: \\ Clinical Ophthalmology \\ 21 March 2017 \\ Number of times this article has been viewed
}

\author{
Francisco José Muñoz \\ Negrete \\ Hans G Lemij ${ }^{2}$ \\ Carl Erb ${ }^{3}$ \\ 'Ophthalmology Service, Hospital \\ Ramón y Cajal, Madrid, Spain; \\ ${ }^{2}$ Glaucoma Service, Rotterdam Eye \\ Hospital, Rotterdam, the Netherlands; \\ ${ }^{3}$ Augenklinik Wittenbergplatz, Berlin, \\ Germany
}

\begin{abstract}
Patient satisfaction with glaucoma treatment has been poorly studied to date. Because glaucoma is a chronic condition in which the therapeutic response is dependent on adherence to treatment, patient acceptability is an important factor in achieving satisfactory outcomes. This multicenter, international (Belgium, the Netherlands, and Spain), epidemiological convenience sample survey among patients commencing treatment with preservative-free latanoprost collected data on patient satisfaction with particular regard to tolerability. A total of 1,541 patients were recruited who were predominantly elderly ( $74 \%$ were over 60 years of age) and female (61\%). Most of the patients had previously received preserved topical glaucoma medication (69\%), 6.7\% had previously received preservative-free medication, whereas $24 \%$ had not previously been treated for glaucoma. The great majority of patients $(>95 \%)$ were satisfied with the preservative-free latanoprost treatment. Among the patients who had previously received preserved medication, $73 \%$ of patients found preservativefree latanoprost to be better tolerated and $89 \%$ found it at least as easy to use as their prior treatment. Patient satisfaction (determined by a $0-100 \mathrm{~mm}$ visual analog scale) was improved by $47 \%$ on a switch from preserved treatment to preservative-free latanoprost. Intraocular pressure was similar in patients who had previously received preserved $(18.3 \mathrm{mmHg})$, preservative-free $(17.8 \mathrm{mmHg})$ glaucoma medication or who were naïve to treatment $(20.3 \mathrm{mmHg})$. Preservative-free latanoprost provided effective reduction of intraocular pressure with better tolerability and patient satisfaction than preserved glaucoma medication. This tolerability profile can be expected to improve adherence to treatment in glaucoma patients.
\end{abstract}

Keywords: prostaglandins analogs, glaucoma therapy, preservative, patient satisfaction, tolerability, persistence, tear substitutes

\section{Introduction}

Glaucoma is a serious, sight-threatening illness that, in the absence of continuing therapy to ameliorate intraocular pressure (IOP), leads inevitably to loss of vision and blindness. Fortunately, effective treatments for reducing IOP are available, and latanoprost eye drops have become the de facto standard of care for most of the glaucoma patients. ${ }^{1}$ However, until recently, latanoprost eye drops have only been available in preservative-containing formulations. The presence of such preservatives in many glaucoma medications is responsible for a range of toxic effects at the ocular surface. ${ }^{2,3}$ Treatment of glaucoma and raised IOP is normally life-long, and in common with other largely symptomless conditions such as hypertension and dyslipidemia, compliance is a major and well-acknowledged problem. ${ }^{4-6}$ Moreover, in the case of glaucoma treatment, compliance may be further compromised by the development of ocular surface disease caused, not by the active component of treatment, but by the preservative used to protect it from bacterial contamination. Indeed, the current iteration of the European Glaucoma Society guidelines makes a strong link between
Negrete

Ophthalmology Service, Hospital

Ramón y Cajal, Ctra de Colmenar Viejo,

km 9, I00, 28034 Madrid, Spain

Tel +34 9l 3368000

Email franciscojmunoz@telefonica.net
Clinical Ophthalmology 2017:I I 557-566

557

Dovepress f in 0

http://dx.doi.org// 0.21 47//OPTH.SI26042 (c) (1) (5) 2017 Muñoz Negrete et al. This work is published and licensed by Dove Medical Press Limited. The full terms of this license are available at https://www.dovepress.com/terms.php

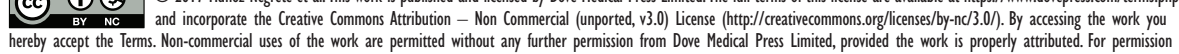
for commercial use of this work, please see paragraphs 4.2 and 5 of our Terms (https://www.dovepress.com/terms.php). 
tolerability and compliance, "a patient who complains about side effects is usually not adherent to therapy".

Preservative-free latanoprost formulations have recently become available, and early indications are that ocular surface symptoms are reduced ${ }^{8}$ and compliance improved., ${ }^{9,10}$ A recent study indicated that, although glaucoma patients generally claimed satisfaction with their treatment, ocular surface disease was widespread. Dissatisfaction with treatment was associated with the presence of ocular signs, symptoms, and the requirement for tear substitutes. ${ }^{11}$ The aim of the present study was to extend these patient satisfaction findings into a group of patients who had recently commenced treatment or been switched from another topical glaucoma treatment to preservative-free latanoprost.

\section{Methods}

The study comprised a multicenter, international, observational survey conducted in the context of routine ophthalmological private practice.

\section{Subjects}

\section{Recruitment}

A representative convenience sample of ophthalmologists in Germany, the Netherlands, and Spain was recruited from databases. Investigators were selected on the basis of feasibility and geographical distribution.

\section{Inclusion criteria}

Patients of either gender, aged at least 18 years with a documented diagnosis of glaucoma, ocular hypertension, visual field defect, or disc abnormalities corresponding to the diagnostic criteria of the European Glaucoma Society Guidelines ${ }^{1}$ and who had received treatment with preservative-free latanoprost (Monoprost ${ }^{\circledR}$ Laboratoires Théa, Clermont Ferrand, France) for at least 3 months (at least 80 days) were eligible for inclusion.

\section{Exclusion criteria}

There were no specific exclusion criteria.

\section{Study plan}

Each recruited ophthalmologist was required to enroll 10 patients within 3 months. Data collection was undertaken between April 2013 and September 2014.

This study involved the use of a two-page questionnaire that was completed at a single visit in the context of a routine consultation for the follow-up of glaucoma treatment. The questionnaire recorded basic demographic information, a brief history of the patient's illness, current and previous treatments, and the reasons for any changes in medication. The ophthalmologists recorded patients' tolerance and satisfaction with their current and previous glaucoma therapy. An ocular examination was undertaken in the course of routine follow-up for glaucoma with particular reference to ocular surface disease.

\section{Evaluation}

\section{Primary endpoint}

The primary variable assessed in the study was the level of satisfaction among preservative-free latanoprost-treated patients expressed in four classes: "Very Satisfied", "Satisfied", "Unsatisfied", and "Very Unsatisfied".

\section{Secondary variables}

The investigator recorded the patient's previous treatments, a brief history of their prior glaucoma treatment and the reasons for any treatment switch. The tolerability of the current glaucoma treatment and of their previous treatment was determined on a $100 \mathrm{~mm}$ visual analog scale (VAS; $0 \mathrm{~mm}$ corresponding to a very low tolerance and $100 \mathrm{~mm}$ to a very high tolerance) by questioning the patient by the physician (Figure 1). Symptoms upon instillation and also between instillations of medication of preservative-free

\section{According to the patient, how is his tolerance to Monoprost ${ }^{\circledR}$ ? \\ Ask the patient to evaluate his/her tolerance on the visual analog scale below. \\ ( 0 corresponding to a very bad tolerance, and 100 very good tolerance)}

Very bad tolerance

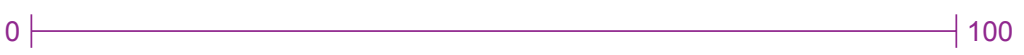

Very good tolerance

Measured value: I_l_ I_ $\mid \mathrm{mm}$ (please use the provided ruler)

How was the tolerance of the previous treatment?

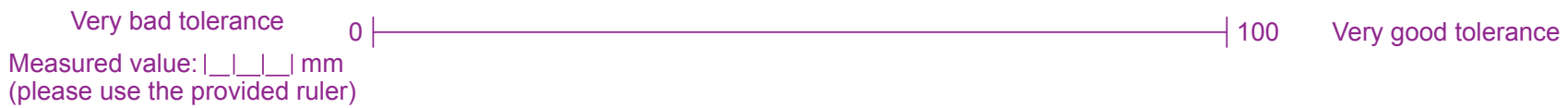

(please use the provided ruler)

Figure I Visual analog scale for determining tolerance to preservative-free latanoprost and previous treatment. 
latanoprost were recorded, as was the frequency of use of any tear substitutes.

An ocular examination was conducted, and eyelid redness and swelling, the presence of eyelid scales or crusts, conjunctival hyperemia, and chemosis were rated on a $0-3$ scale for each eye. Corneal and conjunctival staining and tear breakup time findings, when assessed, were recorded. IOP in each eye of the patients was measured during the visit.

\section{Ethical considerations}

This was a non-interventional observational survey and therefore beyond the scope of the International Conference of Harmonization ( $\mathrm{ICH}$ ). However, issues of autonomy, respect for individual rights, beneficence, non-maleficence, and justice were taken into account according to Good Epidemiological Practice. ${ }^{7}$

Approval was obtained from local or national ethics committees according to the regulations in force in each country and at each investigatory center. The organizations that provided ethical approval are listed in the Supplementary materials.

Patients, having been informed of the purpose of the study and the future of their data, gave signed consent before any data were collected. The data collected were not directly or indirectly nominative, thus approval from data protection organizations was not required.

\section{Statistical methods}

If patients had previously received at least one preserved medication, they were included in the preserved medication group. "Preservative-free" patients were defined as those patients who had previously received only unpreserved topical medication. Treatment naïve patients were defined as those who had received no previous topical treatment before the current preservative-free latanoprost treatment.

Statistical analysis was conducted by using SAS 9.2.

\section{Analysis of variables}

Continuous variables are described in terms of $n$, mean, standard deviation, median, minimum and maximum as appropriate. Categorical variables are provided in terms of absolute frequency and percentage by category. Confidence intervals at $95 \%$ are given where applicable.

All the subjects, having confirmed that they had read the patient information letter, treated at least 80 days with Monoprost $^{\circledR}$ and with a completed case report form were included in the full analysis set.
Logistic regression analysis was used to identify parameters associated with patient satisfaction with their current preservative-free latanoprost treatment. Odds ratios and $P$-values were determined. Descriptive statistics are provided as mean \pm standard deviation and/or range as appropriate.

\section{Results \\ Patient disposition}

A total of 1,541 patients (Germany, 213; Spain, 1,303; the Netherlands, 25) were enrolled.

\section{Demographics}

Demographics and baseline disease information are given in Table 1. Subjects were predominantly elderly $(74.4 \%$ of patients were aged $>60$ years $)$ and female $(61.2 \%$ are female).

\section{Treatment history}

A total of 1,525 patients provided data on their previous treatment; the majority had received preserved treatment $(69.4 \%)$, whereas only $6.7 \%$ had previously received preservative-free topical therapy; and $23.9 \%$ were naïve to treatment. The mean exposure to the current preservativefree latanoprost regimen was 130.4 days \pm 43.94 (range 80-366 days).

\section{Glaucoma history}

The great majority of patients had primary glaucoma, most of whom were in the early stages of their illness $(73.3 \%$ had

Table I Demographic and baseline disease characteristics

\begin{tabular}{ll}
\hline Age, years & 1,523 \\
$\mathrm{n}$ & $66.9 \pm 12.7$ \\
Mean $\pm \mathrm{SD}$ & $21-99$ \\
Range & \\
Sex & 1,535 \\
$\mathrm{n}$ & $61.2 \%$ \\
Female & $38.8 \%$ \\
Male & \\
Glaucoma type & 1,504 \\
$\mathrm{n}$ & $87.2 \%$ \\
Primary & $12.8 \%$ \\
Secondary & \\
Stage of glaucoma & 2,976 eyes \\
$\mathrm{n}$ & $30.3 \%$ \\
Ocular hypertension & $43.0 \%$ \\
Early glaucoma & $17.9 \%$ \\
Moderate glaucoma & $8.7 \%$ \\
Severe glaucoma & 1798 \\
\hline
\end{tabular}

Notes: There were I,54I patients in the overall population. The " $n$ " values in the tables indicate the numbers of patients providing data for each parameter. 
Table 2 Reasons for treatment switch to preservative-free latanoprost among patients previously treated with preserved topical medication $(\mathrm{N}=\mathrm{I}, 018)$

\begin{tabular}{ll}
\hline Reason & Patients (\%) \\
\hline Local intolerance & $650(63.9)$ \\
Insufficient efficacy & $522(51.3)$ \\
Insufficient compliance & $115(11.3)$ \\
Systemic intolerance & $78(7.7)$ \\
Patient's request & $69(6.8)$ \\
Other & $56(5.5 \%)$ \\
\hline
\end{tabular}

either ocular hypertension or early glaucoma). There was a tendency for treatment-naïve patients to have earlier-stage disease (Table 1).

\section{Treatment switches}

Of the 1,135 patients who had previously been treated for glaucoma, the most common reason for switching treatment to preservative-free latanoprost was local intolerance or lack of efficacy (Table 2). Overall, patients switched a mean of 2.4 \pm 1.78 times (range 1-20 times, median 2).

\section{Primary assessment parameter}

Overall, 95.3\% of patients were either satisfied $(57.4 \%)$ or very satisfied $(37.9 \%)$ with the tolerability of their current preservative-free latanoprost treatment. There was little difference between patients previously treated with preserved topical therapy and those previously treated with preservative-free topical therapy (94.8\% and $94.1 \%$ were satisfied or very satisfied with their current preservative-free latanoprost treatment, respectively) (Table 3).

Compared with their previous treatment, most patients (73.4\%) rated preservative-free latanoprost as better $(49.0 \%)$ or much better $(24.4 \%)$ tolerated. A significantly greater proportion of patients who had previously received preserved medication rated preservative-free latanoprost as better or much better tolerated than their previous treatment than did patients previously treated with preservative-free medication (75.1\% vs 57.3\% respectively, $P=0.0005$ ) (Figure 2 ).

\section{Secondary variables \\ IOP}

IOP (left and right eye data merged) was similar in patients who had previously received preserved $(18.3 \pm 4.79 \mathrm{mmHg})$ or preservative-free $(17.8 \pm 4.41 \mathrm{mmHg})$ treatments and in those naïve to treatment $(20.3 \pm 5.31 \mathrm{mmHg})$.

\section{Ease of use}

Preservative-free latanoprost unidoses were considered as easy to use or easier to use than their previous preserved treatment by $89.4 \%$ of patients (Table 4 ).

\section{Tolerability assessed on a VAS}

Patients rated the tolerability of their current preservativefree latanoprost treatment at $82.4 \pm 17.1$ (on a $0-100 \mathrm{~mm}$ VAS), compared to $56.1 \pm 27.3$ for their previous preserved treatment, an overall improvement of $47 \%$. Tolerability to preservative-free latanoprost was better than previous preserved treatment regardless of the specific previous treatment. Figure 3 shows VAS tolerability scores for previous preserved treatments. The switch from preserved treatment to preservative-free latanoprost resulted in notable improvements in tolerability.

\section{Ocular surface disease}

Only 142 of the $1,528(9.3 \%)$ patients who provided data developed ocular surface disease on their current preservativefree latanoprost therapy. Ocular surface disease was mild in $106(74.6 \%)$ cases, moderate in $32(23.9 \%)$, and severe in only $2(1.4 \%)$. Among the patients in whom ocular surface disease developed, the severity was significantly higher in those who had switched from a preserved treatment compared with those who had switched from a preservative-free treatment ( $P=0.0054$, Chi-squared test).

\section{Ocular signs and symptoms}

The majority of patients remained free of eyelid redness, eyelid swelling, lid scales and crusts, chemosis and positive corneal staining. Where these symptoms did develop, the

Table 3 Patient satisfaction according to prior glaucoma treatment

\begin{tabular}{llll}
\hline & All patients & $\begin{array}{l}\text { Previously treated } \\
\text { with preserved } \\
\text { glaucoma medication }\end{array}$ & $\begin{array}{l}\text { Previously treated } \\
\text { with preservative-free } \\
\text { glaucoma medication }\end{array}$ \\
\hline Very satisfied & $37.9 \%(35.4 \%-40.3 \%)$ & $35.8 \%(32.9 \%-38.8 \%)$ & $\begin{array}{l}\text { Patients naïve } \\
\text { to glaucoma } \\
\text { medication }\end{array}$ \\
Satisfied & $57.4 \%(54.9 \%-59.9 \%)$ & $59.0 \%(56.0 \%-62.0 \%)$ & $54.9 \%(44.7 \%-64.8 \%)$ \\
Unsatisfied & $3.9 \%(2.9 \%-4.9 \%)$ & $4.0 \%(2.9 \%-5.4 \%)$ & $5.9 \%(2.2 \%-12.4 \%)$ \\
Very unsatisfied & $0.9 \%(0.5 \%-1.5 \%)$ & $1.1 \%(0.6 \%-2.0 \%)$ & $0 \%$ \\
\hline
\end{tabular}

Note: Figures in parentheses represent the range. 


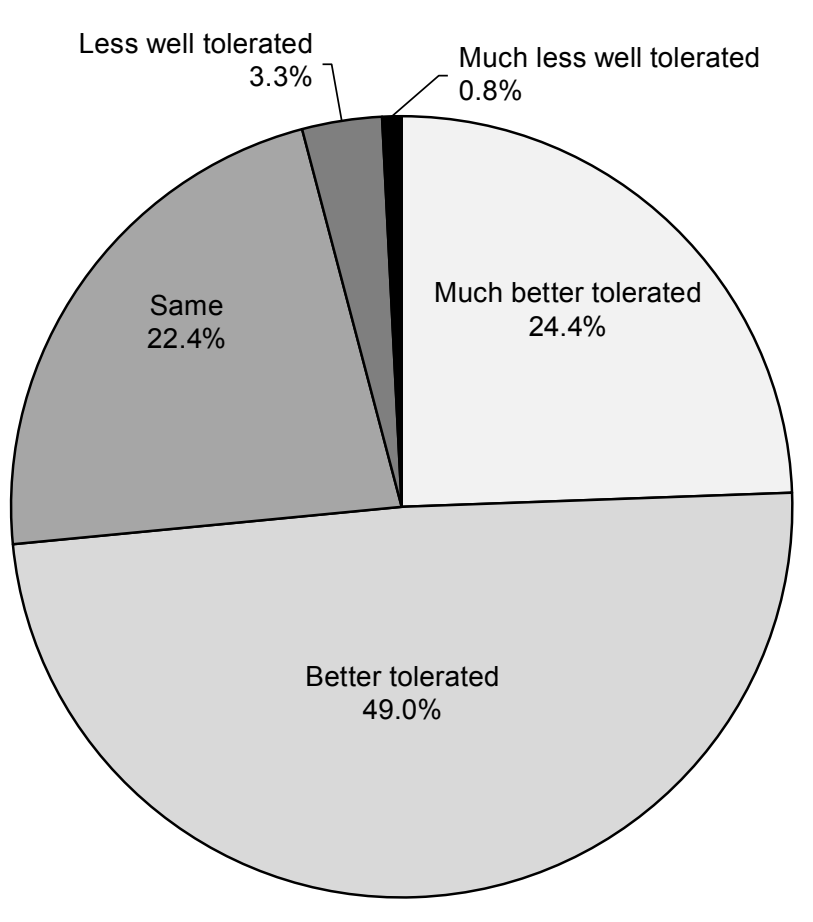

Figure 2 Comparative tolerability of preservative-free latanoprost with previous preserved treatment.

great majority of cases $(>85 \%)$ were of mild severity. Mild hyperemia was the most commonly observed sign (present in $42.6 \%$ of eyes examined), moderate or severe hyperemia was much less common ( $<8 \%$ of eyes examined) (Figure 4$)$.

\section{Use of tear substitutes}

Overall, $45.7 \%$ of patients were using tear substitutes. Use of tear substitute was reduced in preservative-free latanoprost in $28.1 \%$ of patients who had previously been using a preserved treatment.

\section{Association of study parameters with patient satisfaction}

Signs and symptoms of ocular surface disease predicted poorer levels of patient satisfaction; the presence of lid redness (odds ratio 3.22), lid swelling (odds ratio 3.86), lid scales or crusts (odds ratio 2.90), conjunctival hyperemia (odds ratio 2.00), chemosis (odds ratio 3.28), fluorescein-positive corneal staining (odds ratio 2.73), and fluorescein-positive

Table 4 Ease of use of preservative-free latanoprost compared with previous preserved treatement $(n=1,049)$

\begin{tabular}{ll}
\hline & $\%$ patients \\
\hline Much more easy to use & 9.9 \\
More easy to use & 28.9 \\
Same & 50.6 \\
Less easy to use & 10.3 \\
Much less easy to use & 0.3 \\
\hline
\end{tabular}

conjunctival staining (odds ratio 3.13) in both eyes were all associated with a lower likelihood of patient satisfaction with treatment compared with patients who were free of ocular signs.

Treatment naïve patients were approximately twice as likely to be satisfied with treatment compared with previously treated patients (odds ratio 2.0, $P<0.001$ ). The absence of ocular surface disease was strongly associated with satisfaction with treatment (odds ratio 9.8 vs patients with ocular symptoms, $P<0.0001$ ), and patients who found preservativefree latanoprost to be better or much better tolerated than their previous treatment were more likely to be satisfied than those for whom tolerability was the same as their previous treatment (odds ratio 4.5, $P<0.0001$ ). Patients who did not require tear substitutes were more satisfied with treatment than those who did (odds ratio 1.72, $P=0.03$ ), as were those who did not have to increase their use of tear substitutes after switching to preservative-free latanoprost (odds ratio 9.7, $P<0.001)$. Moreover, patients who could decrease the use of tear substitutes were much more likely to be satisfied with treatment than those who needed to increase the use (odds ratio $92, P<0.001)$.

\section{Discussion}

Latanoprost has become the de facto first-line choice for medical therapy for glaucoma. ${ }^{1,12}$ This study concerns a group of patients, the majority of whom were switched from an existing preserved glaucoma treatment to preservativefree latanoprost. The switch to preservative-free latanoprost improved the tolerability of glaucoma therapy. The design of this study has certain limitations; in particular, the sampling method was not fully rigorous leading to the possibility that bias might be present. However, although imperfect, this method does permit reasonably large numbers of patients to be surveyed.

The great majority of patients $(94.7 \%)$ were satisfied with their treatment, regardless of whether they had received prior treatment with preserved or preservative-free topical medications or if they were naïve to glaucoma treatment. Among the patients who switched from another therapy, the great majority found preservative-free latanoprost to be well tolerated as their previous treatment. An effect that was more marked among patients who had previously been receiving a preserved topical therapy. Almost three quarters of patients evaluated preservative-free latanoprost as better tolerated than their previous preserved treatment, and switching from a preserved treatment (regardless of the specific antiglaucoma medication) improved their tolerability by $47 \%$ on the VAS. The use of tear substitutes declined after the 


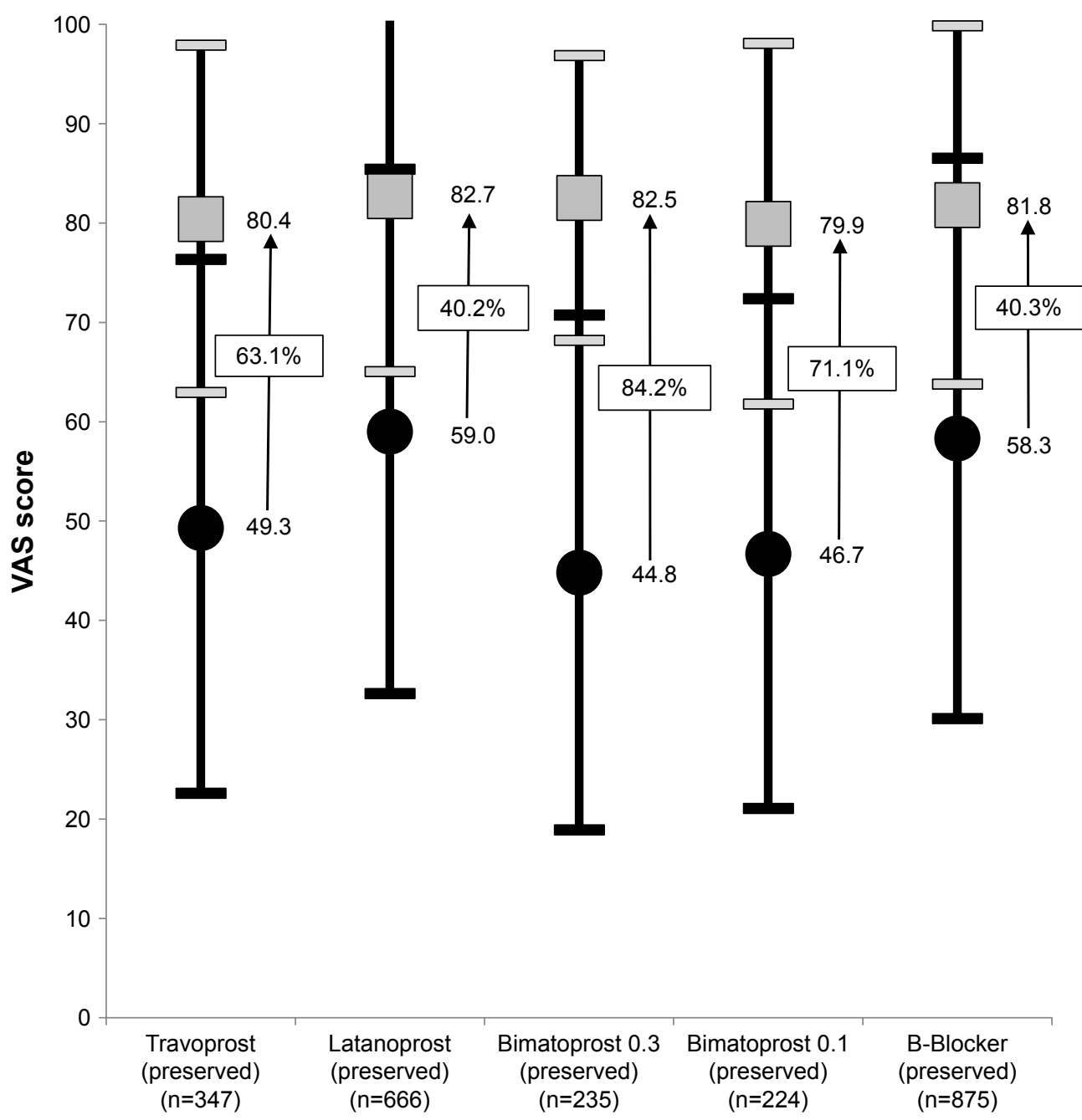

Figure 3 Tolerability of current preservative-free latanoprost treatment compared with previous preserved treatment.

Notes: Figure shows the mean and range of tolerability (as assessed by visual analog scale) of the current preservative-free latanoprost treatment (squares) after switch from the previous preserved treatment (circles). Error bars show the ranges of the mean values.

switch to preservative-free medication. These results are in accord with a recent prospective study showing that glaucoma patients who switched from preserved latanoprost preparation to preservative-free latanoprost retained good control of IOP but benefited from improved tolerability. ${ }^{13}$

The association between ocular surface disease and preservatives used in eyedrops has been well-established in in vivo, in vitro, epidemiological, and clinical studies, and several toxicological mechanisms have been well described. ${ }^{2,3,9}$ Until recently, the presence of such preservatives as benzalkonium chloride were a necessary evil for preventing bacterial contamination of eyedrops. However, preparations are now available that permit the safe deployment of preservative-free eyedrops for the treatment of glaucoma.

Ocular surface disease is common in glaucoma patients, and those displaying symptoms are less likely to adhere to treatment regimens and less likely to be satisfied with their treatment. ${ }^{11,14}$ In the present study, ocular surface disease was present in $<10 \%$ of preservative-free latanoprosttreated patients. Mild hyperemia was, as with all topical prostaglandin analogs, common, but the incidence of moderate and severe hyperemia was low. Ease of use also contributes to persistence with treatment; in the present study, preservative-free latanoprost seemed to be at least as good as patients' prior therapy. ${ }^{15}$ Patient satisfaction (in terms of tolerability) is important in gaining good persistence and compliance with treatment regimens $;{ }^{16}$ patients with better persistence have been shown to have a better-controlled IOP. ${ }^{17}$ A 2-year observational study in 191 patients showed that a significantly greater proportion of glaucoma patients on latanoprost persisted with their treatment for $\geq 24$ months (81.6\%) than those on bimatoprost (22.9\%), travoprost (65.4\%), or timolol (60.5\%). ${ }^{6}$ Glaucoma requires longterm, generally life-long, treatment, and the avoidance of 


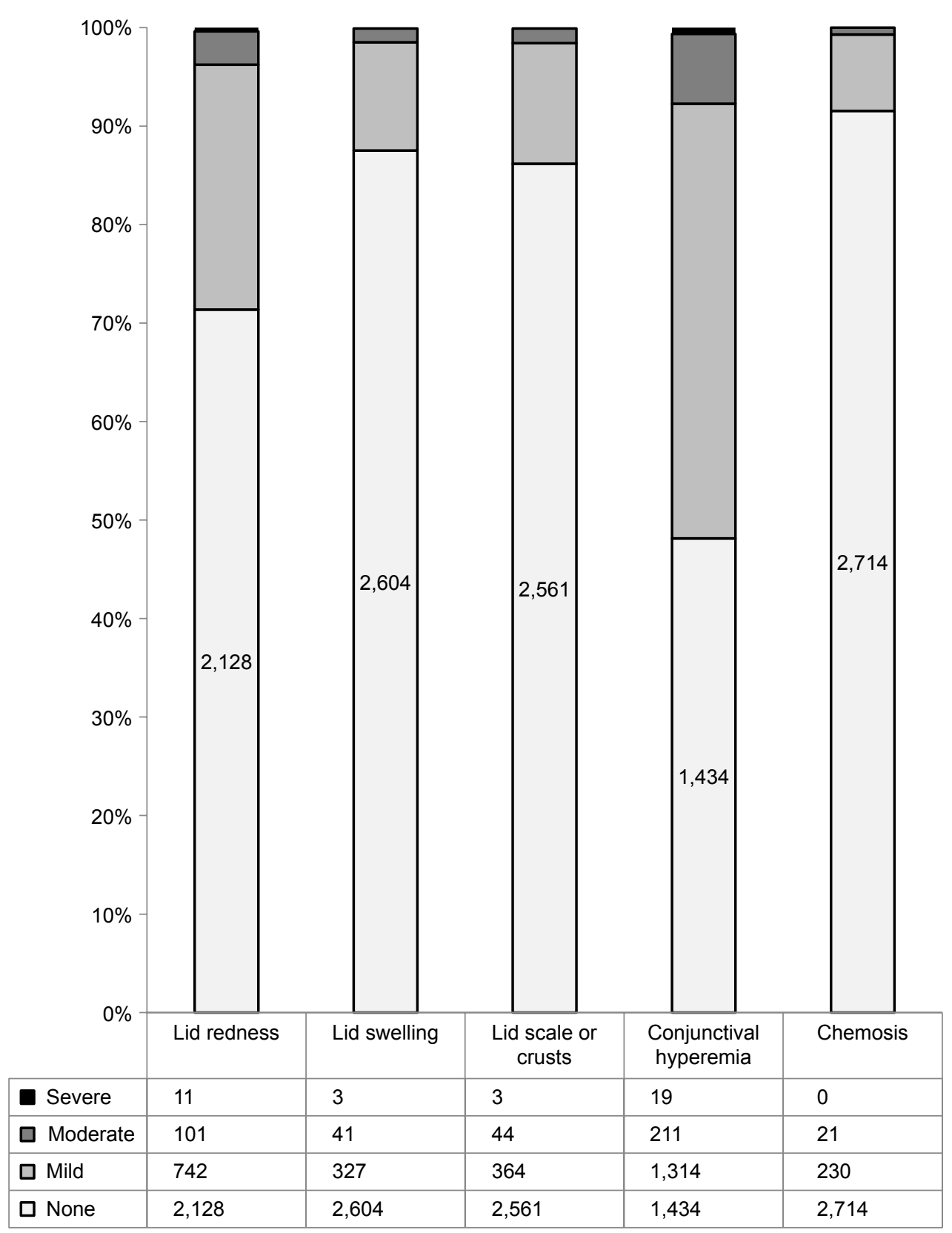

Figure 4 Investigator assessment of the presence of ocular signs $(\mathrm{N}=3,082)$.

ocular surface disease is key to obtaining the adherence and persistence with treatment that will maintain low IOP and preserve patients' vision in the long term.

In the present study, patients seemed to be satisfied with preservative-free latanoprost as their glaucoma medication. Not only this medication was assessed as being better tolerated than their previous preserved medication, but also tolerability was improved by the switch to preservative-free latanoprost. Ease of use was at least as good as the previous medication. Taken together, the preservative-free latanoprost resulted in an effective reduction of IOP with a good tolerability and acceptability profile that could, in general, improve the persistence with anti-glaucoma treatment and thus better preserve vision in the long term.

\section{Disclosure}

This survey was undertaken with funding from Laboratoires Théa under the supervision of the expert group. Members of the expert group were remunerated by Laboratoires Théa for their participation in the study and are consultants to Laboratoires Théa. Editorial assistance in manuscript preparation was provided by Dr JF Stolz who was remunerated 
by Laboratoires Théa. The authors report no other conflicts of interest in this work.

\section{References}

1. European Glaucoma Society Guidelines Fourth Edition. Available from: http://www.eugs.org/eng/EGS_guidelines4.asp. Accessed July 1, 2015.

2. Baudouin C. Detrimental effect of preservatives in eyedrops: implications for the treatment of glaucoma. Acta Ophthalmol. 2008;86(7): 716-726.

3. Baudouin C, Labbé A, Liang H, Pauly A, Brignole-Baudouin F. Preservatives in eyedrops: the good, the bad and the ugly. Prog Retin Eye Res. 2010; 29(4):312-334.

4. Reardon G, Kotak S, Schwartz GF. Objective assessment of compliance and persistence among patients treated for glaucoma and ocular hypertension: a systematic review. Patient Prefer Adherence. 2011;5: 441-463.

5. Yeaw J, Benner JS, Walt JG, Sian S, Smith DB. Comparing adherence and persistence across 6 chronic medication classes. J Manag Care Pharm. 2009;15:728-740.

6. Arias A, Schargel K, Ussa F, Canut MI, Robles AY, Sánchez BM. Patient persistence with first-line antiglaucomatous monotherapy. Clin Ophthalmol. 2010;4:261-267.

7. International Epidemiological Association IEA Good Epidemiological Practice (GEP). Available from: http://ieaweb.org/good-epidemiologicalpractice-gep/. Accessed January 1, 2017.

8. Cucherat M, Stalmans I, Rouland JF. Relative efficacy and safety of preservative-free latanoprost (T2345) for the treatment of openangle glaucoma and ocular hypertension: an adjusted indirect comparison meta-analysis of randomized clinical trials. J Glaucoma. 2014; 23(1):e69-e75.
9. Pisella PJ, Pouliquen P, Baudouin C. Prevalence of ocular symptoms and signs with preserved and preservative free glaucoma medication. Br J Ophthalmol. 2002;86:418-423.

10. Jaenen N, Baudouin C, Pouliquen P, Manni G, Figueiredo A, Zeyen T. Ocular symptoms and signs with preserved and preservative-free glaucoma medications. Eur J Ophthalmol. 2007;17:341-349.

11. Lemij HG, Hoevenaars JG, van der Windt C, Baudouin C. Patient satisfaction with glaucoma therapy: reality or myth? Clin Ophthalmol. 2015;9:785-793.

12. Alm A. Latanoprost in the treatment of glaucoma. Clin Ophthalmol. 2014;8:1967-1985.

13. Rouland JF, Traverso CE, Stalmans I, Fekih LE, Delval L, Renault D, Baudouin C; T2345 Study Group. Efficacy and safety of preservativefree latanoprost eyedrops, compared with BAK-preserved latanoprost in patients with ocular hypertension or glaucoma. Br J Ophthalmol. 2013; 97(2):196-200.

14. Kaštelan S, Tomić M, Metež Soldo K, Salopek-Rabatić J. How ocular surface disease impacts the glaucoma treatment outcome. Biomed Res Int. 2013;2013:696328.

15. Drew T, Wolffsohn JS. Usability of prostaglandin monotherapy eye droppers. Br J Ophthalmol. 2015;99(9):1251-1254.

16. Lee AJ, McCluskey P. Clinical utility and differential effects of prostaglandin analogs in the management of raised intraocular pressure and ocular hypertension. Clin Ophthalmol. 2010;4:741-764.

17. Konstas AG, Maskaleris G, Gratsonidis S, Sardelli C. Compliance and viewpoint of glaucoma patients in Greece. Eye (Lond). 2000;14(Pt 5): $752-756$. 


\section{Supplementary materials List of ethical approval bodies Germany}

- Ethik-Kommission of the Ärztekammer Berlin

\section{The Netherlands}

- Stichting Code Geneesmiddelenreclame

\section{Spain}

Approval was obtained from the individual Comités de Ética en Investigación Clínica (CEIC):

- CEIC Biomédica Provincial de Granada

- CEIC Área 10 - Hospital Universitario de Getafe

- CEIC Área 11 - Hospital Doce de Octubre

- CEIC Área 2 - Hospital Universitario de la Princesa

- CEIC Área 3 - Hospital Universitario Príncipe de Asturias de Alcalá de Henares

- CEIC Área 4 - Hospital Universitario Ramón y Cajal

- CEIC Área 5 - Hospital Universitario La Paz

- CEIC Área 6 - Hospital Universitario Puerta del Hierro de Majadahonda

- CEIC Área 7 - Hospital Clínico San Carlos de Madrid

- CEIC Área 8 - Fundación Hospital Alcorcón

- CEIC Área de Salud de Ávila

- CEIC Área de Salud de Burgos y Soria

- CEIC Área de Salud de León

- CEIC Área de Salud de Salamanca

- CEIC Autonómico de la Rioja

- CEIC Capio Hospital Universitari Sagrat Cor

- CEIC Complejo Hospitalario de Toledo

- CEIC Comunidad Autónoma del País Vasco CEIC-E

- CEIC Consorci Sanitari de Terrassa

- CEIC Consorci Sanitari Integral

- CEIC Consorcio Hospital General Universitari de València

- CEIC Consorcio Hospitalario Provincial de Castellón

- CEIC Corporació Sanitaria Parc Taulí

- CEIC de Aragón-CEICA

- CEIC de Asturias

- CEIC de Cantabria

- CEIC de Galicia

- CEIC de las Islas Baleares

- CEIC de Navarra
- CEIC del Complejo Hospitalario Materno-Infantil Insular de Las Palmas

- CEIC Fundació Catalana d'Hospitals

- CEIC Fundació de Gestió Sanitaria Hospital de la Santa Creu i Sant Pau

- CEIC Fundación Oftalmológica del Mediterráneo

- CEIC Fundacioó Investigacio en Atenció Primaria Jordi Gol i Gorina

- CEIC Hospital Arnau de Vilanova de Valencia

- CEIC Hospital Ciudad de Jaén

- CEIC Hospital Clínic de Barcelona

- CEIC Hospital Clínico de Fuenlabrada

- CEIC Hospital Clínico Universitario de Valencia

- CEIC Hospital Costa del Sol

- CEIC Hospital de Gran Canaria Dr Negrín

- CEIC Hospital de Sagunto i C.E.

- CEIC Hospital General Universitario de Alicante

- CEIC Hospital General Universitario de Elche

- CEIC Hospital General Universitario de Guadalajara

- CEIC Hospital General Universitario Reina Sofía de Murcia

- CEIC Hospital General Universitario San Juan de Alicante

- CEIC Hospital Infanta Cristina

- CEIC Hospital La Fe de Valencia

- CEIC Hospital Torrecárdenas

- CEIC Hospital Universitari Germans Trias i Pujol

- CEIC Hospital Universitario de Canarias

- CEIC Hospital Universitario de Puerto Real

- CEIC Hospital Universitario Dr Peset

- CEIC Hospital Universitario Nuestra Señora de la Candelaria

- CEIC Hospital Universitario Puerta del Mar

- CEIC Hospital Universitario Reina Sofía de Córdoba

- CEIC Hospital Universitario Severo Ochoa de Leganés

- CEIC Hospital Universitario Virgen de la Macarena

- CEIC Hospital Universitario Virgen de la Victoria

- CEIC Instituto de Microcirugia Ocular-IMO

- CEIC Málaga Nordeste - Hospital Regional Universitario Carlos

- CEIC Sevilla Sur - Hospital Universitario de Valme

- CEIC Vissum Corporación Oftalmológica 


\section{Publish your work in this journal}

Clinical Ophthalmology is an international, peer-reviewed journal covering all subspecialties within ophthalmology. Key topics include: Optometry; Visual science; Pharmacology and drug therapy in eye diseases; Basic Sciences; Primary and Secondary eye care; Patient Safety and Quality of Care Improvements. This journal is indexed on

Submit your manuscript here: http://www.dovepress.com/clinical-ophthalmology-journal
PubMed Central and CAS, and is the official journal of The Society of Clinical Ophthalmology (SCO). The manuscript management system is completely online and includes a very quick and fair peer-review system, which is all easy to use. Visit http://www.dovepress.com/ testimonials.php to read real quotes from published authors. 